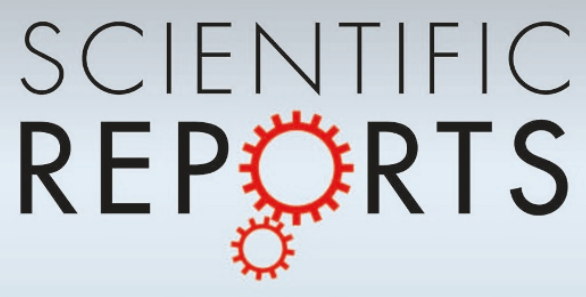

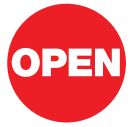

SUBJECT AREAS:

CANCER SCREENING

DIAGNOSTIC MARKERS

BIOMEDICAL ENGINEERING

CANCER

Received

2 October 2012

Accepted

28 January 2013

Published

12 February 2013

Correspondence and requests for materials should be addressed to A.A.S.B. (ali.bhagat@ gmail.com) or C.T.L.

(ctlim@nus.edu.sg)

* These authors contributed equally to this work.

$\dagger$ Current Address: Clearbridge

BioMedics Pte Ltd, Singapore.

\section{Isolation and retrieval of circulating tumor cells using centrifugal forces}

\author{
Han Wei Hou 1,2, Maijid Ebrahimi Warkiani ${ }^{2 *}$, Bee Luan Khoo ${ }^{3 *}$, Zi Rui Lii ${ }^{2}$, Ross A. Soo ${ }^{4,5,6}$, \\ Daniel Shao-Weng Tan ${ }^{7}$, Wan-Teck Lim ${ }^{7}$, Jongyoon Han ${ }^{1,2,8}$, Ali Asgar S. Bhagat ${ }^{2 \dagger} \&$ Chwee Teck Lim ${ }^{2,3,9}$
}

\begin{abstract}
'Department of Electrical Engineering \& Computer Science, Massachusetts Institute of Technology, Cambridge, MA, USA, ${ }^{2}$ BioSystems and Micromechanics (BioSyM) IRG, Singapore-MIT Alliance for Research and Technology (SMART) Centre, Singapore, ${ }^{3}$ Mechanobiology Institute, National University of Singapore, Singapore, ${ }^{4}$ Cancer Science Institute of Singapore, National University of Singapore, Singapore, ${ }^{5}$ Department of Haematology-Oncology, National University Hospital, Singapore, ${ }^{6}$ Department of Surgery, University of Western Australia, Australia, ${ }^{7}$ National Cancer Centre Singapore, Singapore, ${ }^{8}$ Department of Biological Engineering, Massachusetts Institute of Technology, Cambridge, MA, USA, ${ }^{9}$ Department of Bioengineering and Department of Mechanical Engineering, National University of Singapore, Singapore.
\end{abstract}

Presence and frequency of rare circulating tumor cells (CTCs) in bloodstreams of cancer patients are pivotal to early cancer detection and treatment monitoring. Here, we use a spiral microchannel with inherent centrifugal forces for continuous, size-based separation of CTCs from blood (Dean Flow Fractionation $(D F F)$ ) which facilitates easy coupling with conventional downstream biological assays. Device performance was optimized using cancer cell lines ( $>85 \%$ recovery), followed by clinical validation with positive CTCs enumeration in all samples from patients with metastatic lung cancer $(\mathrm{n}=20 ; 5-88 \mathrm{CTCs}$ per $\mathrm{mL})$. The presence of $\mathrm{CD}_{133^{+}}$cells, a phenotypic marker characteristic of stem-like behavior in lung cancer cells was also identified in the isolated subpopulation of CTCs. The spiral biochip identifies and addresses key challenges of the next generation CTCs isolation assay including antibody independent isolation, high sensitivity and throughput $(3 \mathrm{~mL} / \mathrm{hr})$; and single-step retrieval of viable CTCs.

$P$ resence and frequency of circulating tumor cells (CTCs) in bloodstream of patients with epithelial cancers (carcinomas) is an important intermediate step in cancer metastasis and provides valuable insights associated with disease stage ${ }^{1,2}$ and treatment evaluations ${ }^{3}$. As compared to obtaining fresh tissue biopsy which is often technically challenging and causes great inconvenience to patients, "liquid biopsy" for CTCs detection can be carried out routinely in patients due to accessibility and ease of blood collection. More importantly, because primary tumor sampling may not reflect the actual metastatic conditions, CTCs also serve as a representative surrogate tumor biomarker for real-time monitoring of disease status and tailoring personalized therapy $\mathrm{y}^{4,5}$. Despite significant clinical relevance, progress on CTCs research is hindered by the lack of efficient and robust CTCs separation techniques due to their extremely rare occurrence ( $\sim 1-100$ CTCs per $10^{9}$ blood cells). This coupled with their highly heterogeneous morphologies and molecular signatures makes their isolation from blood technically challenging and limits their use as potential cancer biomarkers in clinical cancer management ${ }^{6}$.

Existing macroscale CTCs isolation/enrichment methods include density-gradient centrifugation or RBC lysis to extract mononuclear cells, physical filtration with commercial filter pores (isolation by size of epithelial tumor cells (ISET) $)^{7}$ or immunomagnetic separation against surface molecules commonly expressed on malignant epithelial cells (most notably the commercial CellSearch ${ }^{\circledR}$ system from Veridex) ${ }^{8}$. Long processing time coupled with laborious sample preparations often resulting in cell contamination or low CTCs recovery have hindered the development of these techniques into an routine in vitro diagnostic test. Currently, the CellSearch ${ }^{\circledR}$ test is the only FDA approved test for ascertaining prognosis for patients with metastatic breast, colorectal and prostate cancer. The application of microfluidics based technologies for CTCs separation is an attractive alternative which not only offers better control of the microenvironment during separation but also facilitates integration and automation for high throughput sample processing. Current microfluidic CTCs isolation technologies are primarily based on physical/size based filtration ${ }^{9-11}$ or immuno-mediated CTCs capture in surface functionalized channels or microstructures ${ }^{12-17}$. Although affinity binding methods generally provide better separation purity, the use of surface antigens is less desirable as their expression levels are highly heterogeneous and tumor-dependent and retrieval of captured CTCs is non-trivial due to strong cell binding in the device ${ }^{18}$. Moreover, there is a risk of losing the most aggressive CTCs subpopulation due to epithelial-to-mesenchymal transition (EMT) which leads 
to down-regulation of epithelial markers (e.g. EpCAM) commonly used for affinity binding and may thus underestimate the number of actual CTCs present in the bloodstream ${ }^{19}$.

Microfluidic flows are usually associated with dominant viscous drag forces (low Reynolds number, $R e$ ) which are responsible for laminar flow profiles entraining suspended particles and cells along streamlines ${ }^{20}$. Recently, we and others have shown that particles can migrate across streamlines to focus at distinct positions in microchannels due to significant inertial forces (henceforth known as inertial microfluidics) which can be exploited for high throughput, sizebased particle and cell separation ${ }^{21-24}$. Briefly, particle lateral migration and focusing occurs due to the superposition of two inertial lift forces $\left(\mathrm{F}_{\mathrm{L}}\right)$ acting on the particles in opposite directions: the shearinduced lift force and the wall-induced lift force ${ }^{22}$. In curvilinear channels (for e.g. spiral), particles experience additional lateral Dean drag force $\left(\mathrm{F}_{\mathrm{D}}\right)$ due to presence of transverse Dean flows arising from the centrifugal acceleration of fluid flow in curved channels. Interplay between inertial lift and Dean forces in curvilinear channels can be exploited for an efficient, high resolution size-based separation $^{25,26}$. Although much success has been demonstrated for particles and cell separation ${ }^{24,27}$, the use of inertial microfluidics for bloodrelated separation is greatly limited by the large red blood cells (RBCs) background $(\sim 45 \% v / v)$ whereby cell-cell interactions can severely affect the cell focusing behavior and hence deteriorate separation efficiency ${ }^{28}$. Typically, blood samples have to be diluted significantly $(50-100 \times)$ in inertial microfluidic systems, which increases processing time to the level unsuitable to process large volumes of clinical blood samples required for CTCs isolation applications ${ }^{29}$.

To address these challenges, we introduce an inertial microfluidics-based separation technique for CTCs isolation from blood, aptly termed Dean Flow Fractionation (DFF). Complete separation between a mixture of $7.32 \mu \mathrm{m}$ and $1.9 \mu \mathrm{m}$ polystyrene particles has been previously demonstrated based on similar principle ${ }^{30}$. In curvilinear channels, the influence of centrifugal acceleration in radial direction results in the formation of two symmetrical counter-rotating vortices (top and bottom) across the channel cross-section $^{31}$, also known as Dean vortices. The magnitude of these secondary flows is characterized by a non-dimensional Dean number $(D e)$ and particles flowing in curvilinear channels experience a lateral drag force $\left(F_{D}\right)$ which entrain and drive them along the direction of flow within the Dean vortex ${ }^{30,32}$. This motion translates to the particles moving back and forth along the channel width between the inner and outer walls with increasing downstream distance when viewed from the top or bottom. This lateral migration can be defined in terms of 'Dean cycle' (DC), where a particle, which is initially positioned near the microchannel outer wall, migrates to the inner wall at a given distance downstream is said to have completed $1 / 2$ Dean cycle (DC 0.5). The same particle returning back to the original position near the channel outer wall would complete a full Dean cycle (DC 1). Apart from Dean drag force, larger particles and cells (particle diameter $a_{p} / h>0.07 ; a_{p}$ is the particle/cell diameter) in curvilinear microchannels also experience appreciable inertial lift forces $\left(\mathrm{F}_{\mathrm{L}}\right)$ near the inner wall and the resultant particle focusing position is strongly dependent on the ratio of inertial lift to Dean drag forces $\left(\mathrm{F}_{\mathrm{L}} / \mathrm{F}_{\mathrm{D}}\right)$ which scales exponentially with particle $\operatorname{size}^{25,26,27}$.

By exploiting the size difference between CTCs and hematologic cells (CTCs $\sim 10-20 \mu \mathrm{m}$; RBC $\sim 8 \mu \mathrm{m}$ discoid; leukocytes $\sim 7-$ $12 \mu \mathrm{m})^{7,33}$, we take advantage of these two phenomena, i.e. Dean migration and inertial focusing, to achieve efficient CTCs separation from blood. The microchannel design consists of a 2-inlet, 2-outlet spiral microchannel $(500 \mu \mathrm{m}(w) \times 160 \mu \mathrm{m}(h))$ with a total length of $\sim 10 \mathrm{~cm}$ (Figure 1A). Criterion for particle focusing depends on the shortest channel dimension (microchannel height, $h)^{34}$. In our DFF system, channel dimensions are selected such that only the larger CTCs undergo inertial focusing, while migration of the smaller hematologic cells (RBCs and leukocytes) is solely affected by the Dean drag (i.e. only the CTCs satisfy the $\mathrm{a}_{\mathrm{p}} / h \sim 0.1$ ratio). Blood sample ( $\sim 20 \%$ hematocrit (hct)) is pumped into the outer inlet while sheath fluid $(1 \times \mathrm{PBS})$ is pumped through the inner inlet at a higher flow rate to confine the sample stream near the outer wall. As the sample travels through the channel, all the cells (CTCs and blood cells) initiate migration along the Dean vortex and move towards the inner channel. Near the inner wall, larger cancer cells or CTCs focus tightly as they experience strong inertial lift forces, preventing them from migrating further under the influence of Dean drag, while the smaller blood cells continue flowing along the Dean vortex and travel back towards the outer wall. This allows continuous collection of CTCs at the inner outlet and blood cells (RBCs and leukocytes) are removed from the outer outlet as waste. Since the smaller blood cells are not focused or equilibrated in this microchannel, steric crowding effect is eliminated which enables the processing of extremely high hematocrit blood samples $(\sim 20 \%)$ without affecting separation performance.

A key feature of $D F F$ is the ability to continuously collect viable CTCs allowing easy coupling with convectional 96-well plate for subsequent biological assays. The performance of $D F F$ was validated using cancer cells spiked into whole blood $(>85 \%$ cancer cell recovery achieved), followed by preliminary clinical testing with healthy ( $\mathrm{n}$ $=20)$ and lung cancer patients $(n=20)$ blood samples. Sorted cells were enumerated using fluorescent immuno-staining to determine recovery and subsequently cultured demonstrating high viability. Tumor cells were successfully identified in peripheral blood of all cancer patients $(\sim 5-88$ CTCs per $\mathrm{mL})$ with metastatic disease, clearly highlighting its potential as a robust CTCs detection platform. Unlike other affinity-capture or physical filtration microfluidic devices, issues arising from clogging are eliminated by virtue of the large microchannel dimensions and high flow conditions facilitating processing of very high hematocrit blood samples. As high volume throughput is a key requirement of any rare cell isolation technique, the DFF operating conditions are optimized to process $3 \mathrm{~mL}$ whole blood volume in an hour. To our knowledge, this is the fastest processing speed demonstrated in microfluidics systems for continuous CTCs isolation.

\section{Results}

Dean Cycle characterization. COMSOL simulations (COMSOL Inc, Burlington, MA) were used to study the Dean flow profile in the spiral device at varying $R e$ to determine the flow rate required to achieve a complete Dean cycle (DC 1) migration (Fig. S1). Lateral Dean migration effect along the channel was modeled by applying particle tracking function for fluid elements located at the outer wall region of the inlet at $\mathrm{DC} 1(R e \sim 50)$. Corresponding positions of the tracked streamlines were in excellent agreement with the trajectory of $3 \mu \mathrm{m}$ beads determined experimentally at DC 1 . This was expected as the beads $\left(\mathrm{a}_{\mathrm{p}} / h<0.1\right)$ solely undergo Dean migration within the channel without any influence of inertial forces (Fig. S2). To further confirm the operational flow rates for CTCs separation, $15 \mu \mathrm{m}$ and $6 \mu \mathrm{m}$ fluorescent microbeads were tested individually at DC 1 to mimic cancer cells $(\sim 15-20 \mu \mathrm{m}$ in diameter $)$ and RBCs $(\sim 6-$ $8 \mu \mathrm{m})$ respectively. As expected, there were distinct differences in their flow behavior and focusing position as $6 \mu \mathrm{m}$ beads were successfully transposed from the outer wall towards the inner wall and returned back to the outer wall again (similar to $3 \mu \mathrm{m}$ beads) while $15 \mu \mathrm{m}$ beads undergo strong inertial focusing $\left(\mathrm{a}_{\mathrm{p}} / h \sim 0.1\right)$ near the inner wall at the channel outlet (Fig. S3).

Whole blood processing. RBCs constitute $>99 \%$ of all blood cell components and complete RBCs removal is imperative for efficient and meaningful isolation of rare CTCs. After corroboration of the separation principle using fluorescent microbeads, equilibrium positions for RBCs samples of varying hematocrit (at DC 1) were 

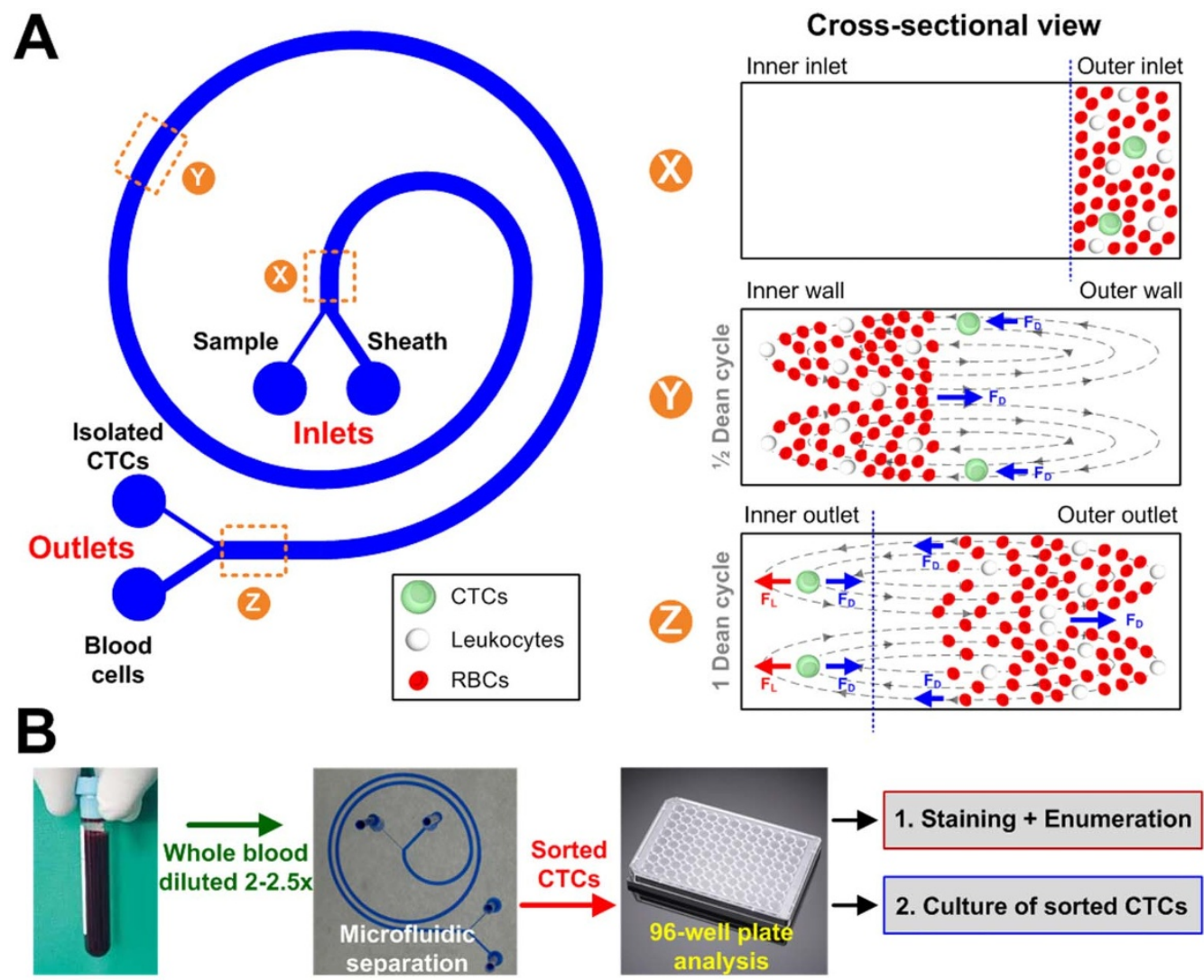

Figure $1 \mid$ (A) Schematic illustration of the separation principle for high-throughput CTCs isolation using Dean Flow Fractionation (DFF). Blood sample and sheath fluid are pumped through the outer and inner inlets of the spiral device respectively. Under the influence of Dean drag forces $\left(\mathrm{F}_{\mathrm{D}}(\right.$ blue arrows)), the smaller hematologic cells (RBCs and leukocytes) migrate along the Dean vortices towards the inner wall, then back to outer wall again (Dean cycle 1), while the larger CTCs experience additional strong inertial lift forces ( $\mathrm{F}_{\mathrm{L}}$ (red arrows)) and focus along the microchannel inner wall, thus achieving separation. (B) Overall workflow of device operation and coupling with 96-well plate for various downstream applications such as CTCs enumeration and culture of sorted CTCs.

used to determine the maximum sample hematocrit that the spiral biochip can handle. Similar to $6 \mu \mathrm{m}$ beads behavior, smaller RBCs experienced negligible inertial forces and were found at the outer half of the channel after a single Dean Cycle migration (Fig. 2A). As hematocrit increased, RBCs band broadened due to cell-cell interaction induced dispersion and a final hematocrit of $20 \%$ was chosen as it gave $\sim 175 \mu \mathrm{m}$ of cell free region at the channel inner wall with negligible RBCs recovery from the CTCs outlet $(150 \mu \mathrm{m})$. Compared to other inertial-based microfluidic separation methods, $20 \%$ hematocrit implies only $\sim 2 \times$ dilution of whole blood (original hematocrit $\sim 40-45 \%$ ). This translates to a processing time of $\sim 20$ minutes for $1 \mathrm{~mL}$ of whole blood after dilution (at $100 \mu \mathrm{L}$ $\left.\min ^{-1}\right)$.

While leukocytes constitute $\sim 0.1 \%$ of all blood cells $(\sim 5$ million per $\mathrm{mL})$, it is still overwhelming when compared to CTCs $(\sim 10$ $100 \mathrm{CTCs} / \mathrm{mL}$ ) and removal of nucleated leukocytes will significantly reduce the background noise for subsequent molecular analysis. To study the depletion capability of leukocytes in the spiral device, a pure population of leukocytes was obtained by RBCs lysis and pumped through the device at DC 1 . High-speed imaging (6400 frames per second) was used to capture their flow positions near the outlet region which can be represented by a linescan of the composite image. As shown in Figure 2B, the larger leukocytes experienced stronger drag forces during the lateral migration and do not migrate completely back to the outer wall at DC 1 . Nevertheless, the cells are still approximately $160 \mu \mathrm{m}$ away from the inner wall which ensures minimal leukocytes contamination in the CTCs outlet. For this reason we fixed the CTC outlet width to $150 \mu \mathrm{m}$.
Cell lines. Figure 2C illustrates the focusing position of MCF-7 cells at different locations along the spiral channel. Due to the small radius of curvature (R) in the first semi-circular spiral loop, larger cancer cells quickly migrated laterally towards the inner wall (position 2) due to strong Dean vortices. With increasing downstream distance, the MCF-7 cells begin to focus tightly near the inner wall as inertial forces have stronger dependence on particle diameter as compared to Dean drag forces $\left(F_{L} \propto a_{p}{ }^{4} v s . F_{D} \propto a_{p}\right)$. By optimizing the width of the CTCs outlet, high cancer cell separation efficiencies $(>85 \%)$ was achieved which was consistent for different cell lines (Fig. 2D) ${ }^{25,30}$. Lastly, multicellular MCF-7 clusters were also tested as CTCs clusters have been reported in clinical samples ${ }^{16,35}$ and may yield important prognostic significance ${ }^{36}$. Despite high flow conditions in our device, separation and collection of intact MCF-7 clusters was successfully achieved. This is due to the short transit time within the channel (highest shear rates) which prevents the breakup of these cell clusters (Fig. 2E).

DFF cascaded spiral system. To demonstrate separation of cancer cells from blood, we spiked a high number of MCF-7 cells $\left(\sim 10^{5} / \mathrm{mL}\right)$ into $20 \%$ hematocrit whole blood samples for testing at DC 1. Following separation, flow cytometry analysis using common surface markers (EpCAM and CD45) was performed on the sorted samples to quantify CTC recovery from other hematologic cellular components. As shown in Figure 3A, focusing positions of MCF-7 cells remained similar in PBS solution and 20\% hematocrit blood samples, clearly confirming that the presence and lateral migration of RBCs did not affect their inertial focusing. For complete RBCs 

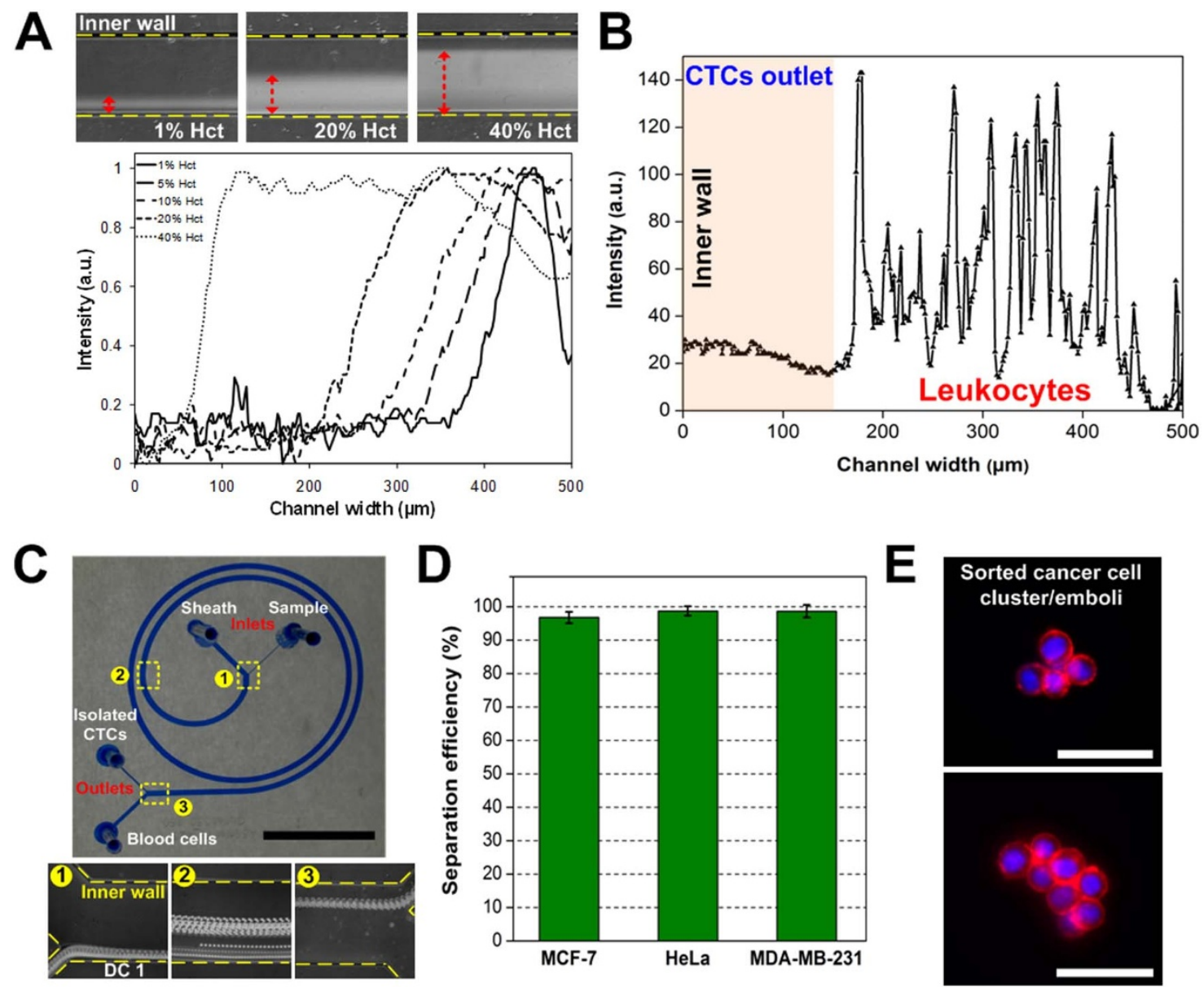

Figure $2 \mid$ Device characterization using hematological cells and cancer cell lines at Dean Cycle 1 (DC 1). (A) Averaged composite images and intensity plot illustrate broadening RBCs occupied regions (red dashed line) for increasing hematocrit prior outlet bifurcation. Yellow dotted lines indicate position of channel walls. (B) Linescan indicating lateral positions of leukocytes near the outlet at DC 1. The larger leukocytes behaved similar to RBCs and were transposed to outer half of the channel under the influence of Dean drag forces, resulting in minimal leukocytes entering the CTCs outlet ( $150 \mu \mathrm{m}$ width). (C) Average composite images representing the focusing position of MCF-7 cells at corresponding locations of the spiral channel. Scale bar is $1 \mathrm{~cm}$. (D) Histogram plot indicating a high separation efficiency of $>85 \%$ for different cancer cell lines tested. (E) Images of sorted MCF-7 clusters stained with anti-EpCAM antibodies (red) and Hoechst dye (blue) to identify the cell membrane and nucleus respectively. Scale bar is $100 \mu \mathrm{m}$.

removal from the sorted CTCs, a 2-stage cascaded spiral system was set up by connecting the CTCs outlet of the first spiral device to the sample inlet of a second spiral device (Fig. S4). This allowed subsequent removal of any residual RBCs and leukocytes which might have entered the CTCs outlet (at the first stage) due to undesirable cell-cell interactions and minimize loss of rare CTCs by eliminating manual transfer of sorted CTCs solution to a new device (Figure 3B). Moreover, throughput and processing time remains the same (20 mins/mL of whole blood) as both spiral devices are operating in conjunction with one another. Based on FACS and hemocytometer analysis, a high cancer cell recovery of $>85 \%$ with enrichment ratio of $10^{9}$ fold (over RBCs) and $\sim 10^{3}$ fold (over leukocytes) was achieved which is better than other size-based and affinity-based CTC sorting techniques typically achieving $10^{4}-10^{6}$ fold overall enrichment. High cell viability $(>98 \%)$ based on Trypan blue assay was also observed (data not shown), and is consistent with others reporting that the high shear conditions in inertial microfluidics have no adverse effects on the sorted cells ${ }^{27}$.

Besides high-throughput processing, DFF also allows continuous collection of sorted CTCs in a single step. Downstream processing such as CTCs enumeration or culture of sorted CTCs can now be readily performed by lab technicians or clinicians with little training and experience required. As an application for CTCs enumeration, we processed $1 \mathrm{~mL}$ of blood samples spiked with MCF-7 cells at physiological CTCs concentrations $(\sim 10-100 / \mathrm{mL})$ followed by immunostaining on the sorted cells. The stained cells were then resuspended in a 96-well plate and enumerated using an inverted epifluorescence microscope equipped with a motorized stage. As shown in Figure 3C (i), MCF-7 cells were easily distinguished from contaminating leukocytes based on fluorescence intensity and cell morphology. High cancer cell recovery $(\sim 85 \%)$ was achieved using small numbers of spiked cancer cells, illustrating minimal cell loss and the potential of our device to process clinically-relevant blood samples (Fig. 3C (ii)). In particular, the high flow rates used in our system prevent settling and non-specific binding of cancer cells in the syringe and tubing, which is an important concern for enumeration of the rare CTCs in blood. Lastly, we demonstrate successful culturing of sorted MCF-7 cells in a 96-well plate (Figure 3C (iii)). Unlike most microfluidic CTCs isolation systems which only allow on-chip growth of CTCs due to difficulties in post-separation retrieval, the combined platform of DFF and 96-well plate potentially simplifies off-chip CTCs culture using standard cell culture techniques. Moreover, the platform offers more flexibility on the culture substrates (culture flasks or membrane) which can also be easily functionalized with different ligands or extracellular matrix (Matrigel or collagen) to provide a more physiological environment for CTCs growth. 

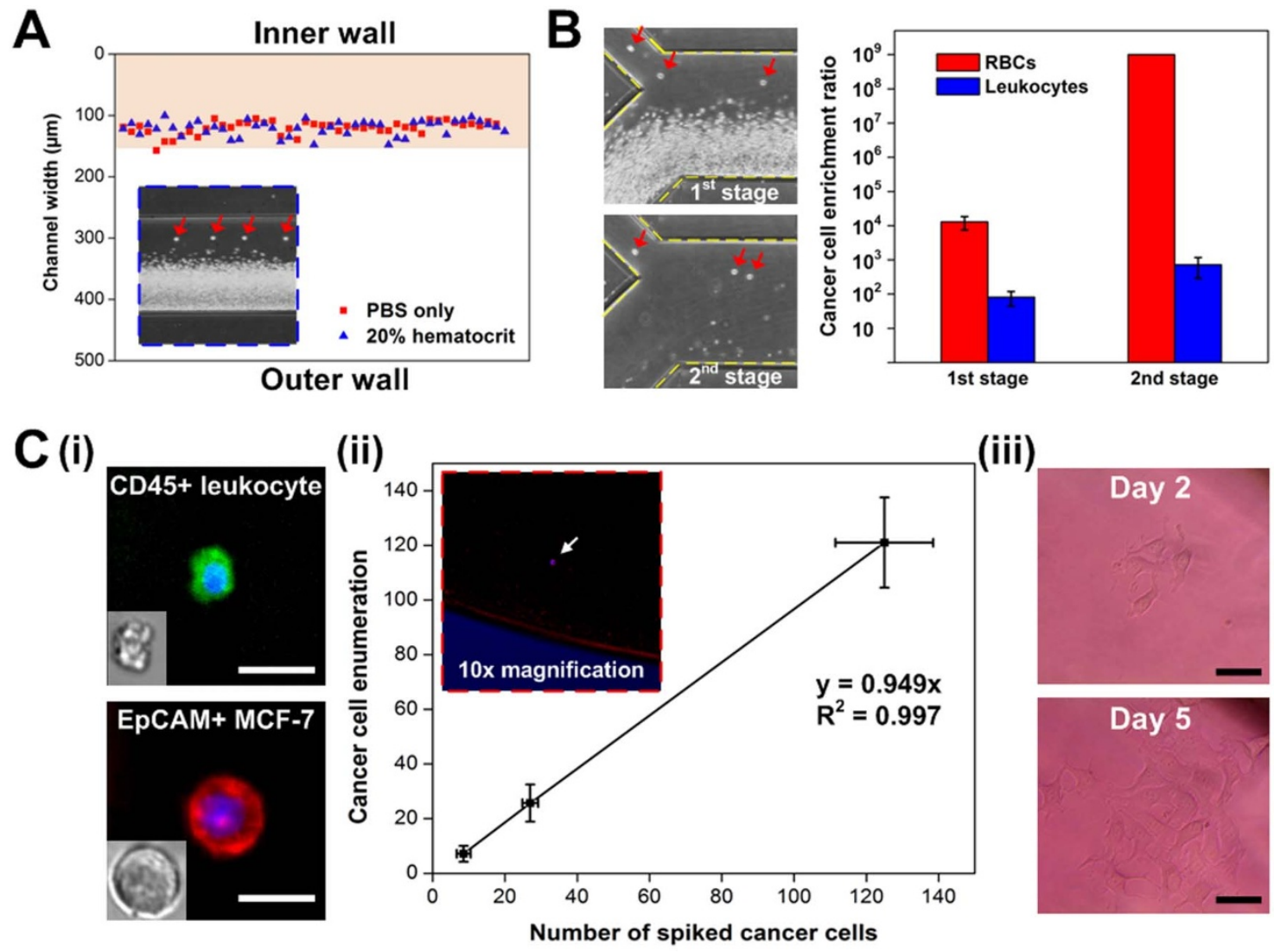

(iii)

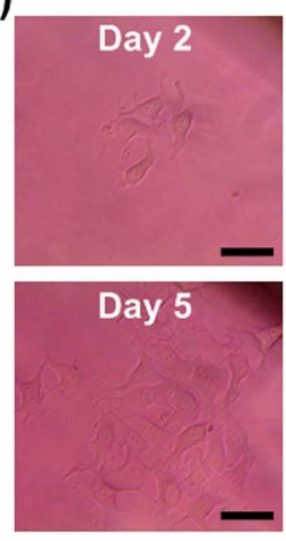

Figure 3 Isolation of cancer cells from blood in a 2-stage cascaded spiral system. (A) Plot indicates similar focusing positions of MCF-7 cells suspended in PBS solution and $20 \%$ hematocrit blood samples within the pink shaded area (150 $\mu \mathrm{m}$ wide) which corresponds to the dimension of CTCs outlet. High speed image (6400 fps) captured at the channel outlet (blue dotted box) clearly illustrates focusing of larger MCF-7 cells at the inner wall (red arrows) while smaller RBCs occupied the outer channel region at DC 1. (B) Representative images highlighting separation of MCF-7 cells (red arrows) from 20\% hematocrit samples at different stages in the cascaded system. Plot (log scale) indicates a high cancer cell enrichment ratio of $10^{9}$ fold (over RBCs) and $\sim 800$ fold (over leukocytes) using the cascaded system. (C) (i) Bright field and fluorescence images of sorted CD45-positive (green) leukocyte and EpCAM-positive (red) MCF-7 cells on 96-well plate after immuno-staining. Both cell types were stained with Hoechst dye for nuclei identification. Scale bar is $20 \mu \mathrm{m}$. (ii) High cancer cell recovery ( 95\%) was achieved using 96-well plate analysis after sorting blood samples spiked with physiological relevant CTCs concentration $(\sim 10-100 / \mathrm{mL}$ ). Inset image (red dotted box) shows a stained cancer cell (white arrow) in a well under $10 \times$ magnification. (iii) Images showing successful culture of sorted MCF-7 cells in 96-well plate. Scale bar is $50 \mu \mathrm{m}$.

Clinical samples testing. After validation of the DFF cascaded system, we processed blood samples ( $\sim 6 \mathrm{~mL}$ volume) from 20 metastatic lung cancer patients at various stages of treatment as an initial clinical proof-of-concept. Blood samples from healthy individuals were also tested as controls $(\mathrm{n}=20)$. After separation, the sample collected from CTCs outlet was spun down and re-suspended in $100 \mu \mathrm{L}$ and the cell suspension was transferred to a 96-well plate for immunofluorescence staining and CTCs enumeration. Cells were stained with Hoechst for DNA content, FITC-conjugated anticytokeratin (CK) antibodies for epithelial cells and APC-conjugated anti-CD45 antibodies for leukocytes. CTCs were enumerated based on CK-positive $\left(\mathrm{CK}^{+}\right)$, CD45-negative $\left(\mathrm{CD} 45^{-}\right)$and Hoechst positive $\left(\right.$ Hoechst $^{+}$) while CD $45^{+}$and Hoechst ${ }^{+}$cells were identified as leukocytes. On the basis of this classification, CTCs were successfully detected in all cancer patients, ranging from 5 to 88 CTCs per $\mathrm{mL}$ $(39.1 \pm 24.8 \mathrm{CTCs} / \mathrm{mL}($ mean \pm s.d. $))$ while 20 healthy subjects had negligible CTCs count $(0.79 \pm 0.42 \mathrm{CTCs} / \mathrm{mL})$ (Fig. $4 \mathrm{~A}, 4 \mathrm{~B}$, see Table S1 and S2 for clinical characteristics). Leukocyte contamination was minimal with $441 \pm 319.5$ leukocytes/mL which corresponds to $\sim 2000$ fold leukocyte reduction and a much improved CTCs/leukocytes purity of $\sim 10 \%$ (sufficient for molecular analysis). The diameter range of CTCs isolated using DFF was also highly heterogeneous among patients (average: $\sim 16 \mu \mathrm{m}$ ) and varies from 10-22.5 $\mu \mathrm{m}$ within the same sample. Although the ability to isolate a large size range of CTCs is favorable, the exact mechanisms responsible for inertial focusing of smaller CTCs $(\sim 10 \mu \mathrm{m})$ remain unclear and warrant further investigation. We hypothesize that the deformability of CTCs might have some secondary effects on CTCs inertial focusing. Interestingly, besides single cell CTCs, we also observed multicellular CTCs cluster present in one of the cancer patient blood samples (Fig. 4C). Unlike single CTCs, the CTCs in these CTC clusters were smaller and approximately the same size as surrounding leukocytes which is consistent with recent report by Cho et al. ${ }^{37}$. The presence and biological significance of such CTCs clusters in blood are still not well understood and it is debatable whether the cells aggregate during circulation or these CTC aggregates break away from the primary tumor as an aggregate and circulate throughout the body ${ }^{35}$. Determining the mechanism behind CTC aggregation is critical in understanding whether they play a role in metastasis and is a topic of active research. In patients with advanced lung cancer, we also stained the CTCs isolated using the spiral biochip with CD133, a phenotypic surface marker for lung cancer cells with higher tumorigenic potential ${ }^{38,39}$. As shown in Fig. 4D, few isolated CTCs $\left(\mathrm{CK}^{+}\right.$, Hoechst $\left.^{+}\right)$were stained positive for CD133 marker, highlighting the capability of our technique to potentially identify and isolate cancer stem cells (CSCs). These cells play a pivotal role in cancer metastasis due to their ability to proliferate and differentiate into multiple cell types. 
A

\begin{tabular}{|c|c|c|c|c|c|c|}
\hline \multirow{3}{*}{ Subjects } & \multirow{3}{*}{$\begin{array}{c}\text { Number of } \\
\text { samples }\end{array}$} & \multicolumn{4}{|c|}{ Range of CTCs/mL } & \multirow{3}{*}{$\begin{array}{c}\text { Samples with } \\
>5 \text { CTCs } / \mathrm{mL} \\
(\%)\end{array}$} \\
\hline & & $0-5$ & $5-20$ & $20-50$ & $50-100$ & \\
\hline & & \multicolumn{4}{|c|}{ Number of samples (\%) } & \\
\hline Healthy (control) & 20 & $20(100)$ & 0 & 0 & 0 & 0 \\
\hline Lung cancer & 20 & $0(0)$ & $5(25)$ & $6(30)$ & $9(45)$ & 100 \\
\hline
\end{tabular}

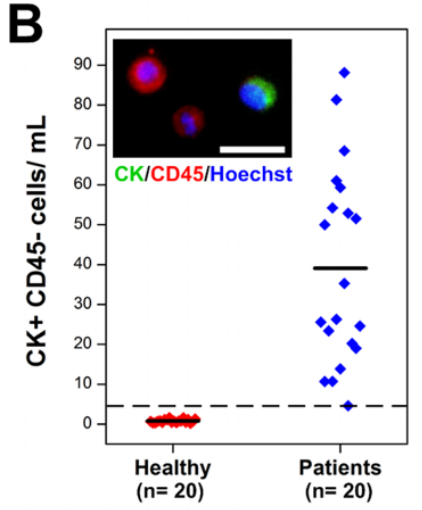

D

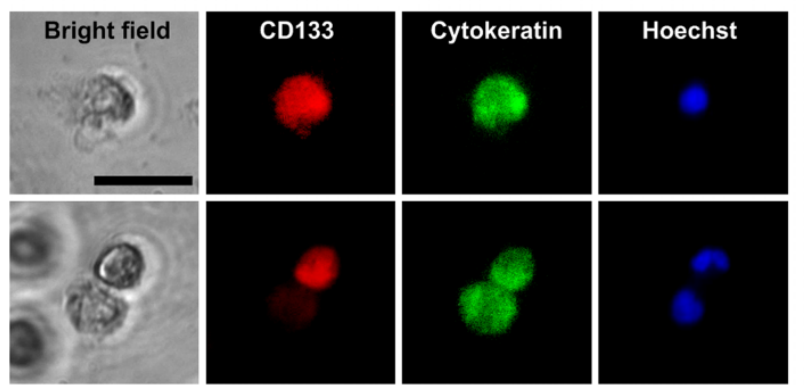

Figure $4 \mid$ Enumeration of CTCs from cancer patients. (A) Summary of CTCs count per $\mathrm{mL}$ of blood for healthy samples and patients with metastatic lung cancer. (B) CTCs enumeration plot for healthy donors (red) and lung cancer patients (blue). Dashed line indicates threshold of $5 \mathrm{CTCs} / \mathrm{mL}$. (Inset) Representative image ( $60 \times$ magnification) of isolated CTCs and leukocytes from lung cancer patients stained with antibodies against cytokeratin (green), CD45 (red) and Hoechst (blue). CTC morphology is characterized by high nuclear: cytoplasmic ratios. Scale bar is $20 \mu \mathrm{m}$. (c ) Optical images $(60 \times$ magnification) of an isolated CTC microcluster using DFF. Scale bar is $50 \mu \mathrm{m}$. (D) Bright field and fluorescence images $(60 \times$ magnification $)$ of sorted CTCs stained for stem cell marker CD133 (red), cytokeratin (green) and Hoechst (blue). Scale bar is $20 \mu \mathrm{m}$.

\section{Discussion}

The CTCs Grand Challenge proposed by Dr. den Toonder recently ${ }^{40}$ listed handling of large fluid volume and collection of viable CTCs after separation as two key unresolved issues of current CTCs separation methods. Although existing inertial microfluidics-based devices allow high throughout processing with good separation resolution, a major limitation is the use of diluted blood samples $(\sim 0.1-$ $2 \%$ hematocrit) which make them unsuitable to process milliliters of clinical blood samples typically required for CTCs separation. In this work, we introduce a high throughput blood separation technique termed as "Dean Flow Fractionation (DFF)" and applied it successfully for CTCs isolation from blood. The use of an additional sheath buffer in our spiral device facilitates the Dean migration of large volume of RBCs in a well-controlled manner, thus allowing us to process high hematocrit blood samples ( $\sim 20-25 \%)$ which is demonstrated for the first time in inertial microfluidics based systems. This translates to a sample processing speed of $\sim 3 \mathrm{~mL} / \mathrm{hr}$ and the large channel dimensions also eliminate clogging issues within the device, ensuring a robust separation platform for processing of large clinical sample volumes.
Numerous microfluidic devices for CTCs separation from blood have been reported in recent years but few have been validated with clinical patient samples. Nagrath et al. developed a CTC-chip consisting of an array of EpCAM-coated microposts and demonstrated 99\% CTC detection in 116 patients with different cancer types ${ }^{12}$. High capture purity $(\sim 50 \%)$ was achieved in their device with a sample flow rate of 1-2 mL/hr. The same group later developed another microfluidic device known as the Herringbone-chip (Hbchip) which eliminates the use of microposts by generating microvortices within the device to promote CTCs binding to EpCAM functionalized surfaces ${ }^{16}$. Clinical validation was shown with successful CTCs detection in 14 out of 15 (93\%) patients with prostate cancer at a higher throughput $(1.5-2.5 \mathrm{~mL} / \mathrm{hr})$ and the authors also demonstrated on-chip cell lysis, followed by RT-PCR for molecular characterization of captured CTCs. Such EpCAM-based microfluidic technologies are particularly advantageous for CTCs enumeration due to high capture purity and there is minimal manual handling of these rare cells as immuno-staining and CTCs detection are done on-chip with a small imaging area to facilitate counting. However, these unique features also inherently limit their ability to couple with conventional biological assays or platforms for other downstream applications (such as CTCs growth or high-throughput drug screening) as retrieval of captured CTCs would be difficult due to strong cell binding to the functionalized substrates. In this aspect, $D F F$ is a more versatile and convenient separation technique as it allows a continuous collection of sorted CTCs in a single step which can be further processed for applications beyond enumeration.

A growing amount of evidence has shown that subpopulation of carcinoma cells at the primary tumor undergo EMT, resulting in their increased motility which facilitates their intravasation into the blood circulation to form CTCs ${ }^{41}$. More recently, EMT has been associated with stem-like phenotype $e^{42,43}$ and this subpopulation of cancer cells, also known as cancer stem cells (CSCs) or tumor initiating cells, are the most resistant to chemotherapy and thus regarded highly important for personalized medicine research ${ }^{44}$. As EMT leads to down-regulation of epithelial markers (e.g. EpCAM) commonly used for CTCs capture in affinity-based methods, systems such as CellSearch ${ }^{\circledR}$ may tend to isolate lower number of CTCs, missing cells that have undergone complete EMT phenotype or have low level of cytokeratin expression ${ }^{45,46}$. Moreover, clinical studies have shown that some CTCs isolated from peripheral blood also express EMT and stem cell markers ${ }^{47,48}$, which further advocates the importance of using non-EpCAM enrichment methods and additional EMT markers such as vimentin and $\mathrm{N}$-cadherin for accurate CTCs enumeration. We successfully showed the existence of $\mathrm{CD}_{133^{+}}$cells in the isolated subpopulation of CTCs in clinical samples from advanced metastatic lung cancer patients. CD133, a surface glycoprotein which has been shown to overexpress in highly tumorigenic cells is a popular phenotypic marker for lung cancer cells with stem-like behavior ${ }^{38,39}$. Significant numbers of $\mathrm{CK}^{-} / \mathrm{CD} 45^{-} / \mathrm{Hoe}-$ chst $^{+}$cells were also collected from the spiral biochip (data not presented) and further molecular testing and sequencing of these rare CTCs subtype and unknown cell population is necessary to determine their origin and their role in cancer metastasis. Although our spiral biochip has the ability to isolate a large heterogeneity of CTC size including CTCs which have overlapping sizes with the leukocytes, the minimum CTC size cutoff is $\sim 11 \mu \mathrm{m}\left(\mathrm{a}_{\mathrm{p}} / \mathrm{h} \sim 0.07\right)$ and CTCs smaller than $10 \mu \mathrm{m}$ are inevitably lost in the waste outlet. This affects the accuracy of the CTCs enumeration but our size-based separation approach would also provide a larger CTCs pool (independent of EpCAM expression) for identification of CTCs subpopulation or EMT-derived CTCs. In addition, CTCs microemboli as large as $50-100 \mu \mathrm{m}$ were also successfully isolated in some cancer patients and the continuous collection mode of our technique potentially allows further molecular studies on these rare cell clusters to gain valuable insights related to cancer metastasis. 
Isolation of these rare CTC is only the first step towards a more comprehensive study and analysis of the role of these cells in the spread of cancer. To this end, we believe the DFF technique offers several distinct advantages over other microfluidic CTCs separation methods. Firstly, the device can process $3 \mathrm{~mL}$ of whole blood in an hour with $100 \%$ CTCs detection in all cancer patients, offering unprecedented throughput and separation performance. Unlike affinity-capture or physical filtration microdevices, the continuous collection of sorted CTCs and short residence time in the device $(<10 \mathrm{msec})$ significantly shortens the CTCs exposure time to constant shear in the channel, thus minimizing any undesirable shearinduced changes to the CTCs phenotype. This is important because retrieval of isolated CTCs for subsequent molecular characterization of biological functions or gene expression for targeted drug therapy is the hallmark of personalized medicine. Lastly, sample preparation is minimal and only syringe pumps are required which simplifies the device setup and operation. This is a key consideration for successful commercialization and implementation in clinical settings as technicians and clinicians can readily run the biochip with little training and experience required. We envision that with all these aforementioned advantages, $D F F$ would be a versatile blood separation technique which can be applied for other diseased cell separations from blood characterized by differences in cell morphology.

\section{Methods}

Device fabrication. The microfluidic devices were fabricated in polydimethylsiloxane (PDMS) using standard microfabrication soft-lithographic techniques described previously ${ }^{49}$. Briefly, the patterned silicon wafers were silanized with trichloro $(1 \mathrm{H}, 1 \mathrm{H}, 2 \mathrm{H}, 2 \mathrm{H}$-perfluorooctyl) silane (Sigma Aldrich, USA and PDMS prepolymer mixed in $10: 1(\mathrm{w} / \mathrm{w})$ ratio with curing agent was poured onto the silanized wafer and baked at $80^{\circ} \mathrm{C}$ for 1-2 hrs. The cured PDMS mold was then used as a template (negative replica) for subsequent PDMS casting which gave the final PDMS microchannels. Holes $(1.5 \mathrm{~mm})$ for fluidic inlets and outlets were punched and the PDMS devices were irreversibly bonded to microscopic glass slides using an air plasma machine (Harrick Plasma Cleaner, USA).

COMSOL modeling. Commercially available COMSOL Multiphysics (COMSOL Inc, Burlington, MA) software was used to study fluid flow behavior in the proposed spiral design. The model structure consists of an inner semi-circular channel with radius $\mathrm{R}_{1}=0.42 \mathrm{~cm}$ and a spiral channel $\mathrm{R}_{2}=(1.0+0.1 \theta / 2 \pi) \mathrm{cm}$. Width $(w)$ and height $(h)$ of the channel were fixed at $500 \mu \mathrm{m}$ and $155 \mu \mathrm{m}$ respectively. Full NavierStokes equations for incompressible fluids were solved using finite element method. For simplicity, it was assumed that the presence of suspended particles does not affect the intrinsic properties of the fluid (a continuum fluid with density $1 \mathrm{~g} \mathrm{~cm}^{-3}$ and dynamic viscosity 0.001 Pa s). Fluid flow with varying flow rates (in $\mathrm{mL} / \mathrm{min}$ ) and noslip boundary conditions were applied at the channel inlet and channel wall boundaries respectively. At the outlet, the pressure was set to zero with no viscous stress on the boundary. As the flow is symmetric about the middle plane in the $\mathrm{z}$ direction (depth), only half of the channel (upper channel) was modeled (Figure S1), which introduced an additional symmetric boundary condition for that symmetric plane. From the steady-state solution for single-phase laminar flow, Dean migration of the fluid elements (similar as particles) at the outer wall region were obtained using particle tracking functionality in the software and compared with experimental observations of fluorescence microbeads lateral migration. For calculation of Dean flow, a separate semi-circular channel with the same dimensions as the spiral channel was modeled under similar fluid flow conditions. The radius of the channel was set as $0.42 \mathrm{~cm}$ and $1.0 \mathrm{~cm}$ respectively, which corresponds to inner and outer loop of the spiral channel. Maximum Dean and axial flow speed were then calculated at mid point along the circular channel at different flow rates, where the error caused by the boundary effect is expected to be negligible.

Cell culture. Human breast adenocarcinoma cell lines, MCF-7 and MDA-MB-231, and human cervical HeLa cell line were used to mimic CTCs separation in this work. The cells were cultured in low-glucose Dulbecco's modified Eagle's medium (DMEM) (Invitrogen, USA) supplemented with 10\% fetal bovine serum (FBS) (Invitrogen, USA) together with $1 \%$ penicillin/streptomycin (Invitrogen, USA). The culture was maintained at $37^{\circ} \mathrm{C}$ in a humidified atmosphere containing $5 \%(\mathrm{v} / \mathrm{v}) \mathrm{CO}_{2}$. The cells were sub-cultivated every 4 days with media replaced every 48 hours. Sub-confluent monolayers were dissociated using $0.01 \%$ trypsin and $5.3 \mathrm{mM}$ EDTA solution (Lonza, Switzerland).

Sample preparation. Fluorescently labeled microbeads of 6 and $15 \mu \mathrm{m}$ diameter (Fluoresbrite ${ }^{\circledR}$ Microspheres, Polysciences Inc, Singapore) were added $(0.01 \%$ volume fraction) to sample buffer which consists of $1 \times$ phosphate buffered saline (PBS), $2 \mathrm{mM}$ ethylenediaminetetraacetic acid (EDTA) supplemented with $0.5 \%$ bovine serum albumin (BSA) (Miltenyi Biotec, Germany). BSA was used to prevent non-specific adsorption to the tubing and microchannel walls. For RBC hematocrit experiments, whole blood obtained from healthy donors was spun down to separate the RBCs. Final sample concentration was adjusted to varying hematocrit $(1 \%-45 \%)$ with sample buffer accordingly. For leukocyte control experiments, whole blood was treated with RBC lysis buffer (eBioscience, USA) according to the manufacturer's instructions to obtain a pure population of leukocytes. To study the focusing of cancer cells in spiral channel, a high concentration of cancer cells $\left(\sim 10^{5}\right.$ cells $\left./ \mathrm{mL}\right)$ were added into the sample buffer to facilitate high speed imaging and analysis. Whole blood experiments were carried out by diluting fresh blood samples to $\sim 20 \%$ hematocrit spiked with MCF-7 cells $\left(\sim 10^{5} / \mathrm{mL}\right)$. $>100000$ events of cells for flow cytometry (FACS) analysis were recorded for greater accuracy of data obtained.

Device characterization. During testing, cancer cell and blood sample was filled in a $1 \mathrm{~mL}$ syringe and pumped through the spiral microfluidic device using a syringe pump (NE-1000, New Era Pump Systems Inc., USA) while sheath flow $(1 \times$ PBS, $2 \mathrm{mM}$ EDTA supplemented with $0.5 \%$ BSA) was filled in a $60 \mathrm{~mL}$ syringe and pumped into the device using a separate syringe pump (PHD 2000, Harvard Apparatus, USA). The flow rate ratio between the sample and sheath flow was fixed at 1: 9 to form a tight sample stream at the outer wall. The devices were mounted on an inverted phase contrast microscope (Olympus IX71) equipped with a high speed CCD camera (Phantom v9, Vision Research Inc., USA). High speed videos were captured at the channel outlet using Phantom Camera Control software and then analyzed using Image ${ }^{\circledR}$ software. For fluorescence imaging, another inverted microscope (Olympus IX81, Olympus Inc., USA) equipped with a 12-bit EMCCD camera (iXon ${ }^{\mathrm{EM}}+885$, Andor Technology, USA) was used. Images were acquired using Metamorph ${ }^{\circledR}$ software (Molecular Devices, USA) and analyzed using Image $J^{\circledR}$ software.

Immunofluorescence staining and FACS analysis. To determine the enrichment ratio between the sample and sorted CTCs, flow cytometry (FACS) analysis using $\mathrm{BD}^{\mathrm{TM}}$ LSR II flow cytometer (BD Biosciences, USA) was performed on the inlet and CTC outlet samples. Immunofluorescence staining allowed differentiating the various cell types for visualization and quantification. After sorting, the cells were stained with allophycocyanin (APC) conjugated Epithelial Cell Adhesion Molecule (EpCAM) (1:100, Miltenyi Biotec Asia Pacific, Singapore) and fluorescein isothiocyanate (FITC) conjugated CD45 marker (1: 100, Miltenyi Biotec Asia Pacific, Singapore) for 30 minutes to identify MCF-7 cells and blood leukocytes respectively. RBCs concentration was further confirmed using hemocytometer measurement.

For clinical samples, cells isolated from the spiral biochip were fixed and permeabilized using the Inside Stain Kit (MiltenyiBiotec Asia Pacific, Singapore) according to the manufacturer's protocol. CTCs were identified by staining with FITC-conjugated pan-cytokeratin (CK) (1:100, MiltenyiBiotec Asia Pacific, Singapore) for 30 minutes at $4^{\circ} \mathrm{C}$ and washed twice with $1 \times$ PBS before imaging. Cells staining positive for pan-CK and Hoechst (nuclei stain) and negative for CD45 with characteristic morphology of cancer cells (i.e., high nucleus to cytoplasm ratio) are identified as CTCs. Cells staining positively for CD45 and Hoechst and negatively for pan-cytokeratin are identified as leukocytes. APC-conjugated CD133 (1:100, MiltenyiBiotec Asia Pacific, Singapore) was also used for tumor subtype identification in patients with advanced lung cancer.

Sample preparation and clinical testing of study subjects. Blood samples were obtained from healthy volunteers and metastatic lung cancer patients with informed consent. The clinical sample collection protocols were reviewed and approved by the Domain Specific Review Boards of the National University Health System, Singapore, National University of Singapore and the Singapore Health Services (SingHealth). A total of 20 blood samples from healthy volunteers were used as controls and 20 samples from lung cancer patients were processed for CTC enumeration. Clinicopathologic information was recorded for all patients. All blood specimens were collected in EDTA-contained vacutainer tubes (BD, Franklin Lakes, NJ, USA) and diluted $1: 2(1 \mathrm{X})$ with PBS buffer prior to processing on chip. The CTC capture from actual cancer patient samples was performed in a similar procedure to that described above. An average of $6 \mathrm{~mL}$ blood volume was processed for lung cancer patient samples while $3 \mathrm{~mL}$ blood volume was processed from healthy volunteers as control (Supplementary Table 1 and 2).

1. Budd, G. T. et al. Circulating tumor cells versus imaging - Predicting overall survival in metastatic breast cancer. Clin. Cancer Res. 12, 6403-6409 (2006).

2. Cristofanilli, M. et al. Circulating Tumor Cells, Disease Progression, and Survival in Metastatic Breast Cancer. N Engl J Med 351, 781-791 (2004).

3. Hayes, D. F. et al. Circulating tumor cells at each follow-up time point during therapy of metastatic breast cancer patients predict progression-free and overall survival. Clin. Cancer Res. 12, 4218-4224 (2006).

4. Punnoose, E. A. et al. Molecular Biomarker Analyses Using Circulating Tumor Cells. PLoS ONE 5, e12517 (2010).

5. Maheswaran, S. et al. Detection of Mutations in EGFR in Circulating Lung-Cancer Cells. New England Journal of Medicine 359, 366-377 (2008).

6. Pantel, K., Brakenhoff, R. H. \& Brandt, B. Detection, clinical relevance and specific biological properties of disseminating tumour cells. Nat. Rev. Cancer 8, 329-340 (2008). 
7. Vona, G. et al. Isolation by size of epithelial tumor cells - A new method for the immunomorphological and molecular characterization of circulating tumor cells. Am. J. Pathol. 156, 57-63 (2000).

8. Riethdorf, S. et al. Detection of Circulating Tumor Cells in Peripheral Blood of Patients with Metastatic Breast Cancer: A Validation Study of the CellSearch System. Clin. Cancer Res. 13, 920-928 (2007).

9. Mohamed, H., Murray, M., Turner, J. N. \& Caggana, M. Isolation of tumor cells using size and deformation. J. Chromatogr. A 1216, 8289-8295 (2009).

10. Zheng, S. et al. Membrane microfilter device for selective capture, electrolysis and genomic analysis of human circulating tumor cells. J. Chromatogr. A 1162 154-161 (2007).

11. Tan, S., Yobas, L., Lee, G., Ong, C. \& Lim, C. Microdevice for the isolation and enumeration of cancer cells from blood. Biomedical Microdevices 11, 883-892 (2009).

12. Nagrath, S. et al. Isolation of rare circulating tumour cells in cancer patients by microchip technology. Nature 450, 1235-1239 (2007).

13. Adams, A. A. et al. Highly efficient circulating tumor cell isolation from whole blood and label-free enumeration using polymer-based microfluidics with an integrated conductivity sensor. J. Am. Chem. Soc. 130, 8633-8641 (2008).

14. Gleghorn, J. P. et al. Capture of circulating tumor cells from whole blood of prostate cancer patients using geometrically enhanced differential immunocapture (GEDI) and a prostate-specific antibody. Lab Chip 10, 27-29 (2010).

15. Xu, Y. et al. Aptamer-Based Microfluidic Device for Enrichment, Sorting, and Detection of Multiple Cancer Cells. Anal. Chem. 81, 7436-7442 (2009).

16. Stott, S. L. et al. Isolation of circulating tumor cells using a microvortex-generating herringbone-chip. Proceedings of the National Academy of Sciences 107, 18392-18397 (2010).

17. Saliba, A.-E. et al. Microfluidic sorting and multimodal typing of cancer cells in self-assembled magnetic arrays. Proceedings of the National Academy of Sciences 107, 14524-14529 (2010).

18. Spizzo, G. et al. High Ep-CAM Expression is Associated with Poor Prognosis in Node-positive Breast Cancer. Breast Cancer Research and Treatment 86, 207-213 (2004).

19. Pecot, C. V. et al. A Novel Platform for Detection of $\mathrm{CK}^{+}$and $\mathrm{CK}^{-}$CTCs. Cancer Discovery 1, 580-586 (2011).

20. Squires, T. M. \& Quake, S. R. Microfluidics: Fluid physics at the nanoliter scale. Rev. Mod. Phys. 77, 977-1026 (2005).

21. Seo, J., Lean, M. H. \& Kole, A. Membrane-free microfiltration by asymmetric inertial migration. Appl. Phys. Lett. 91, 3 (2007)

22. Di Carlo, D., Irimia, D., Tompkins, R. G. \& Toner, M. Continuous inertial focusing, ordering, and separation of particles in microchannels. Proc. Natl. Acad. Sci. U. S. A. 104, 18892-18897 (2007)

23. Di Carlo, D. Inertial microfluidics. Lab Chip 9, 3038-3046 (2009).

24. Bhagat, A. A. S., Hou, H. W., Li, L. D., Han, J. Y. \& Lim, C. T. Pinched flow coupled shear-modulated inertial microfluidics for high-throughput rare blood cell separation. Lab Chip 11, 1870-1878 (2011).

25. Kuntaegowdanahalli, S. S., Bhagat, A. A. S., Kumar, G. \& Papautsky, I. Inertial microfluidics for continuous particle separation in spiral microchannels. $L a b$ Chip 9, 2973-2980 (2009).

26. Russom, A. et al. Differential inertial focusing of particles in curved low-aspectratio microchannels. New J. Phys. 11, 9 (2009).

27. Lee, W. C. et al. High-throughput cell cycle synchronization using inertial forces in spiral microchannels. Lab Chip 11, 1359-1367 (2011).

28. Hou, H. W. et al. Microfluidic Devices for Blood Fractionation. Micromachines 2 , 319-343 (2011).

29. Hur, S. C., Mach, A. J. \& Carlo, D. D. High-throughput size-based rare cel enrichment using microscale vortices. Biomicrofluidics 5, 022206 (2011).

30. Bhagat, A. A. S., Kuntaegowdanahalli, S. S. \& Papautsky, I. Continuous particle separation in spiral microchannels using dean flows and differential migration. Lab Chip 8, 1906-1914 (2008).

31. Dean, W. The stream-line motion of fluid in a curved pipe. Philosophical Magazine Series 7 5, 673-695 (1928)

32. Ookawara, S., Street, D. \& Ogawa, K. Numerical study on development of particle concentration profiles in a curved microchannel. Chemical Engineering Science 61, 3714-3724 (2006).

33. Zabaglo, L. et al. Cell filtration-laser scanning cytometry for the characterisation of circulating breast cancer cells. Cytometry Part A 55, 102-108 (2003).

34. Bhagat, A. A. S., Kuntaegowdanahalli, S. S. \& Papautsky, I. Enhanced particle filtration in straight microchannels using shear-modulated inertial migration. Physics of Fluids 20, 101702 (2008).
35. Cho, E. H. et al. Characterization of circulating tumor cell aggregates identified in patients with epithelial tumors. Physical Biology 9, 016001 (2012).

36. Hou, J.-M. et al. Clinical Significance and Molecular Characteristics of Circulating Tumor Cells and Circulating Tumor Microemboli in Patients With Small-Cell Lung Cancer. Journal of Clinical Oncology 30, 525-532 (2012).

37. Edward, H. C. et al. Characterization of circulating tumor cell aggregates identified in patients with epithelial tumors. Physical Biology 9, 016001 (2012).

38. Bertolini, G. et al. Highly tumorigenic lung cancer CD133+ cells display stem-like features and are spared by cisplatin treatment. Proceedings of the National Academy of Sciences 106, 16281-16286 (2009)

39. Eramo, A. et al. Identification and expansion of the tumorigenic lung cancer stem cell population. Cell Death Differ 15, 504-514 (2007).

40. den Toonder, J. Circulating tumor cells: the Grand Challenge. Lab Chip 11, 375-377 (2011)

41. Lee, J. M., Dedhar, S., Kalluri, R. \& Thompson, E. W. The epithelial-mesenchymal transition: new insights in signaling, development, and disease. J. Cell Biol. 172, 973-981 (2006)

42. Mani, S. A. et al. The Epithelial-Mesenchymal Transition Generates Cells with Properties of Stem Cells. Cell 133, 704-715 (2008).

43. Al-Hajj, M., Wicha, M. S., Benito-Hernandez, A., Morrison, S. J. \& Clarke, M. F. Prospective identification of tumorigenic breast cancer cells. Proceedings of the National Academy of Sciences 100, 3983-3988 (2003).

44. Li, X. et al. Intrinsic Resistance of Tumorigenic Breast Cancer Cells to Chemotherapy. Journal of the National Cancer Institute 100, 672-679 (2008).

45. Lin, H. K. et al. Portable Filter-Based Microdevice for Detection and Characterization of Circulating Tumor Cells. Clin. Cancer Res. 16, 5011-5018 (2010).

46. Marrinucci, D. et al. Fluid biopsy in patients with metastatic prostate, pancreatic and breast cancers. Physical Biology 9, 016003 (2012).

47. Theodoropoulos, P. A. et al. Circulating tumor cells with a putative stem cell phenotype in peripheral blood of patients with breast cancer. Cancer Letters 288, 99-106 (2010).

48. Aktas, B. et al. Stem cell and epithelial-mesenchymal transition markers are frequently overexpressed in circulating tumor cells of metastatic breast cancer patients. Breast Cancer Research 11, R46 (2009)

49. Hou, H. W. et al. Deformability based cell margination-A simple microfluidic design for malaria-infected erythrocyte separation. Lab on a Chip 10, 2605-2613 (2010)

\section{Acknowledgements}

This research was supported by the National Research Foundation Singapore through the Singapore MIT Alliance for Research and Technology's BioSystems and Micromechanics Inter-Disciplinary Research programme. This work is also supported by the use of MIT's Microsystems Technology Laboratories for wafer fabrication and the lab facilities at the Mechanobiology Institute and the Nano Biomechanics Laboratory at the National University of Singapore. The clinical samples and data collection was supported by Singapore National Medical Research Council grant NMRC 1225/2009. The authors thank Leon D. Li (MIT) for wafer fabrication during the early part of this work

\section{Author contributions}

H.W.H., A.A.B., J.H. and C.T.L. designed research, H.W.H., A.A.B., M.E.W. and B.L.K. performed experiments and analyzed the data, Z.R.L helped with COMSOL modeling. R.A.S., D.S.W.T. and W.T.L. contributed regents and clinical samples. H.W.H., A.A.B., J.H. and C.T.L. wrote the manuscript. All authors reviewed the manuscript.

\section{Additional information}

Supplementary information accompanies this paper at http://www.nature.com/ scientificreports

Competing financial interests: H.W.H., A.A.B., J.H. and C.T.L., along with others, have filed a patent application on the technology described here.

License: This work is licensed under a Creative Commons

Attribution-NonCommercial-NoDerivs 3.0 Unported License. To view a copy of this license, visit http://creativecommons.org/licenses/by-nc-nd/3.0/

How to cite this article: Hou, H.W. et al. Isolation and retrieval of circulating tumor cells using centrifugal forces. Sci. Rep. 3, 1259; DOI:10.1038/srep01259 (2013). 